\title{
LA VOCACIÓN PROFESIONAL, LOS VALORES Y EL PROYECTO DE VIDA DE UN GRUPO DE MILLENNIALS UNIVERSITARIOS DEL PERÚ
}

\author{
Professional calling, values and life projects from a group of \\ peruvian university millenials students
}

\author{
Aymé Gabriela Buitrón Aranda \\ Universidad Peruana Cayetano Heredia \\ ayme.buitron@upch.pe \\ Luz Carbajal Arroyo \\ Universidad Peruana Cayetano Heredia \\ luz@upch.pe
}

Fecha de Recepción:

05 de setiembre de 2017

Fecha de Aceptación:

28 de octubre de 2017

\section{RESUMEN}

El objetivo de la presente investigación fue conocer el proyecto de vida y la escala de valores de los estudiantes de la Universidad Peruana Cayetano Heredia, que expresan mayor interés en trabajar sólo en la profesión que estudian. La muestra estuvo conformada por estudiantes de Biología y Farmacia, se eligieron los que cursaban el cuarto año de estudios porque se asumió que ya contaban con una personalidad definida.

Se halló que existe una significación estadistica importante entre: la vocación que expresan y la clase social media o alta de pertenencia; también entre la vocación y la poca o mediana valoración que le dan a tener pareja e hijos.

Los estudiantes de biología y farmacia muestran características que favorecen su desarrollo profesional y de investigador. Se les puede ubicar dentro de los llamados Millenials, pero no están de acuerdo con el aborto ni se muestran materialistas en sus elecciones.

Palabras clave: vocación, proyecto de vida, estudiantes universitarios, millennials, cultura juvenil

\section{ABSTRACT}

Our goal is to find out about the Cayetano Heredia 's college students life projects and their scale of values, those which reveal more interest to work only on the profession they picked to study, as the biology and pharmacy students from where we chose students from the fourth academic year as their personalities are more clearly defined.

We found that there is an important and significant statistic between the calling they reveal and the social class they belong to, as well as middle as high; as well as how much they value or they would like to stablish relationships or having children.

Pharmacy and biology students show features that agree with their professional development and as researchers. Such ones can be placed among those called Millenials, but they disagree with abortion and do not show their selves as materialists.

Keywords: calling, life project, college students, millennials, youth culture 


\section{INTRODUCCIÓN}

Vamos a estudiar el proyecto de vida y escala de valores de los estudiantes de la Universidad Peruana Cayetano Heredia (UPCH), con énfasis en los alumnos que manifiestan mayor interés en trabajar sólo en la profesión que estudian, en comparación a otros estudiantes de la misma universidad; es así que se eligió estudiar a los jóvenes que cursaban el cuarto año, porque se consideró que ya poseían una personalidad definida.

El interés de ejercer la profesión se vincula directamente a la vocación y esta se desarrolla en la socialización de la persona dentro de una familia y estructura social; el crecimiento individual depende del sistema de valores que aprende en su familia y otras instituciones de la sociedad. Los valores son inherentes a la estructura y al funcionamiento de las instituciones sociales, dentro de las cuales los individuos se desarrollan. Es en este sentido que nos interesa conocer el perfil personal de los estudiantes de la UPCH, en los siguientes aspectos:

1. Los datos sociodemográficos del entrevistado

2. El proyecto de vida y valoración de la vida familiar

3. La escala de valores y metas

Es importante conocer el perfil social y aspectos de la personalidad de los jóvenes universitarios con mayor vocación por la profesión elegida, ya que en esa medida será posible darles una mejor educación, tanto en el hogar como en las instituciones educativas para enrumbar a los jóvenes en la consecución de sus fines en la vida, dentro de un contexto académico.

Parsons (1951) señala que a partir de su personalidad el individuo actúa motivado por sus necesidades y la búsqueda de la gratificación, para lo cual parte de las expectativas que le despiertan las personas con las que interactúa y evalúa las alternativas a su alcance. A partir de esto podemos entender que en el estudio de la vocación se debe analizar los aspectos de la personalidad vinculados a la elección profesional, los valores y las metas vinculados a la realización personal dentro de la sociedad.
Respecto a las personas y su vocación, algunos estudios nos indican que ellos pueden tener personalidades similares y solucionarán problemas del mismo modo. La vocación es un proceso dinámico que se desarrolla con el crecimiento del individuo y con el cambio de las estructuras sociales (Pantoja, 1992). Así cuando la educación es altamente valorada en una sociedad, el sistema educativo ocupa una parte importante de la vida de las personas.

La perspectiva funcionalista analiza las formas en que los valores funcionan en la cultura y la estructura social, para mantener la sociedad como un todo. Los valores reflejan distintas soluciones que las sociedades desarrollan ante los problemas de regulación de la actividad de sus miembros, así como las relativas a la organización y justificación de las prioridades de ellos.

En los planes de estudio del sistema educativo actual, ya se está incluyendo la formación en valores, sobre todo en las carreras vinculadas a la salud y su cuidado. En una época de crisis de valores nos preguntamos cómo estos cambios influyen en los estudiantes y docentes, en general en la institución universitaria, por esto debemos acercarnos a su conocimiento.

Los valores culturales que caracterizan a una sociedad se infieren estudiando las rutinas diarias, las metas de vida, los métodos típicos de aprendizaje formal e informal, los sistemas legales, las actividades de entretenimiento entre otras. Los valores predominantes de las sociedades también han sido inferidos mediante el estudio de los valores de los individuos, esta perspectiva contempla los valores individuales, en parte como producto de una cultura compartida y en parte como resultado de la experiencia única de cada individuo. La media de las prioridades valorativas de los miembros de una sociedad refleja los valores comunes de la cultura. La variación individual en torno a esta media es el reflejo de la personalidad y experiencia única de las personas (Triandis, 1994).

La teoría axiológica de Schwartz (1999) sostiene que los valores se derivan de tres requerimientos universales humanos: las necesidades del organismo, las motivaciones sociales o de interacción y las 
demandas institucionales para la supervivencia de los grupos. La vocación la podemos ubicar como un tipo de necesidad que busca gratificación en la realización profesional, asociada a las motivaciones sociales y en segundo lugar a las demandas institucionales.

Según Maslow (1954) existe una tipología y jerarquía de valores en la medida que existe una jerarquía de necesidades. En dicha tipología, la jerarquía de valores y de necesidades son interdependientes y para que aparezcan los del nivel superior, deben estar satisfechos y realizados los de auto conservación o básicos, siendo éstos últimos de carácter universal. Los valores están basados en una concepción motivacional que se relaciona con la evolución y cumplimiento de las potencialidades de los seres humanos. Por lo que podemos plantear que las necesidades básicas o del organismo y otras vinculadas a la supervivencia, deben verse satisfechas para dar lugar a otras como la vocación.

Un aspecto importante en el desarrollo de la persona en la actualidad es la inclinación que tienen algunos por un individualismo exacerbado en la competencia dentro y fuera de las aulas, presentándose así un conflicto entre los intereses $\mathrm{o}$ valores individuales y los valores colectivos. $\mathrm{La}$ búsqueda del logro individual puede entrar en conflicto con el valor de la benevolencia y el valor de la cooperación en el grupo, más aún si pensamos en la calidad de líder que debe tener un profesional, en tanto ellos son un grupo privilegiado de nuestra sociedad, en los cuáles recae parte importante del desarrollo del país.

Triandis (1994) encuentra que la mayoría de las conductas individualistas ocurren cuando la persona está sola o en pareja, en cambio las conductas colectivistas se dan en pequeños grupos, de culturas colectivistas. La competencia es más frecuente en las culturas de tipo individualista, lo cual es propio de nuestra sociedad actual.

Triandis (1994) reconoce variantes en el individualismo, ya que en función del grado de libertad que se experimenta se construye un yo independiente, pero no necesariamente diferente a los demás miembros de su cultura; en el colectivismo, se contempla una alta igualdad y baja libertad, con un yo interdependiente y compartido con los demás. Los colectivistas se caracterizan por su sentido de sacrificio para el beneficio del grupo y su vocación por cumplir las obligaciones impuestas por las normas sociales. El trabajo de Ciochina y Faria (2011) nos explica cómo el ser colectivista promueve el desarrollo intelectual, en tanto se considera que los demás pueden contribuir a tu progreso intelectual, es decir se asocia a una concepción dinámica de la inteligencia, en la que se promueve el aprendizaje en la experiencia de interacción del grupo.

El estudio de los valores también es importante para conocer cómo mejorar la salud mental de las personas, es así como en el trabajo de Alicia Omar (2006) se explica cómo los valores colectivistas contribuyen en el desarrollo de mejores formas de afrontar el estrés.

Es relevante comprender el estilo de vida al que se asocian los valores y las prácticas culturales de la vida diaria, para acercamos a los proyectos de vida de los jóvenes. El estilo de vida está muy unido al tipo de trabajo que realizamos y también al entorno cultural vinculado a los medios de comunicación, las actividades artísticas, la literatura, el teatro, la música y el cine.

Respecto al estilo de vida de los jóvenes que estamos estudiando, tenemos los estudios sobre la generación "Millennials", Penagos y Rubio (2015) explican las diferencias generacionales de la siguiente manera:

a. La generación de mayor edad es denominada los "Baby Boomers", nacidos entre 1945 y 1964. Ellos se caracterizan por aspirar a construir una carrera extraordinaria, donde el reconocimiento llega a través del dinero, la posición laboral y el estatus (Molinari, 2011).

b. La denominada generación "X" son los nacidos entre 1964 y 1980 . Ellos se caracterizan por ser desconfiados e individualistas. Los " $\mathrm{X}$ " creen menos en el matrimonio, en los gobiernos y en los políticos; varios de ellos vieron cómo las organizaciones no fueron fieles a sus padres y por eso no depositan su futuro en una sola empresa 
(Molinari, 2011). La familia es todavía un valor como generación y se muestran abiertos a la diversidad sexual, de raza y política.

c. La generación "Y" o Millennials son los jóvenes nacidos entre 1981 y 2000 (Molinari, 2011), ellos tienen características parecidas a los de la generación "X" (Begazo y Fernández Baca, 2015). Según el Centro Nacional de Planeamiento Estratégico (2015) en los siguientes 10 o 15 años la población económicamente activa del Perú estará formada en un $60 \%$ por los llamados Millennials, ya que serán alrededor de 7 millones de personas. Los Millennials no se sienten cómodos con los horarios de oficina, ellos son grandes seguidores de las nuevas tecnologías y buscan formar sus propias empresas (Begazo y Fernández Baca, 2015).

En Argentina caracterizan a los Millennials como personas que provienen de una niñez sobresaturada de actividades y horarios, que se han educado en una cultura servidor cliente que ha marcado sus vidas y su estilo de consumo. (Cuesta, Ibáñez, Tagliabue y Zangarro, 2009). La felicidad de estos jóvenes descansa principalmente en vivir de lo que les gusta, viajar mucho y conocer distintos tipos de lugares y personas. (Barómetro- opinión pública e investigación de mercado, 2013). Los Millennials argentinos señalan en un 35\% que su meta en la vida es formar una familia y tener buenas amistades, el $33.8 \%$ señala que su meta es vivir muchas experiencias de vida y el $31.2 \%$ señala que lo más importante es alcanzar una buena posición económica, desarrollarse profesionalmente y destacar o ser exitoso (Cuesta, Ibáñez, Tagliabue y Zangaro, 2009).

A nivel mundial el 91\% considera que el dinero es primordial a la hora de buscar empleo y la misma proporción considera de igual importancia la estabilidad (Emailingnetwork, 2014).

En España la mitad de los Millennials que están casados tienen hijos, pero cada vez más personas los tienen fuera del marco legal del matrimonio. El $43 \%$ de Millennials sigue viviendo con sus padres y el $67 \%$ de quienes se han independizado, lo hacen para vivir con su pareja. Los Millennials españoles se preocupan mucho por el empleo, el acceso a la vivienda y el poder adquisitivo y el $70 \%$ busca realizarse como persona.
Es en este contexto que buscaremos comprender a nuestra población de estudio, ya que el desarrollar una vocación y ejercer la profesión, implica en la actualidad, desarrollarse como persona en el contexto antes descrito.

\section{MÉTODO}

\section{Diseño y plan de análisis}

Se realizó un análisis exploratorio de los datos. El análisis es descriptivo, mediante tablas de frecuencias simples y para las variables principales se desarrollan comparativamente tablas de doble entrada.

Una vez terminada las encuestas, se revisaron y se procedió al ingreso de los mismos al programa Excel y luego fueron analizados utilizando el programa STATA v12.

Para comparar las respuestas sobre valoración se utilizó la prueba de chi cuadrado o prueba exacta de Fisher (si hay alguna frecuencia esperada $<5$ ), con una significancia estadística de 0.05. Asimismo, se calcularon los intervalos al $95 \%$ de confianza para las proporciones respecto a cada una de las variables.

\section{Instrumentos y materiales}

La encuesta fue elaborada por las investigadoras y se aplicó a los estudiantes seleccionados para el estudio. La encuesta fue totalmente anónima y voluntaria, aplicada sólo a estudiantes de 18 años o más.

El instrumento se validó a través de dos expertos del tema a nivel internacional. Los aspectos que validar fueron los siguientes:

Ordenamiento lógico de las preguntas y pertinencia de las alternativas de respuesta.

Pertinencia del enunciado, comprensión y extensión de las preguntas y alternativas de respuestas, así como sugerencias al respecto.

Correspondencia entre los objetivos del estudio y las preguntas del cuestionario. 
Sugerencia de preguntas pertinentes de acuerdo con el título y objetivo de estudio.

\section{Población y Muestra}

La población de estudio comprende a los estudiantes de 4to año de estudios de todas las Facultades de la Universidad Peruana Cayetano Heredia a excepción de los estudiantes de Medicina Humana. Dicha universidad es privada y se encuentra en Lima, la capital del Perú, esta población corresponde aproximadamente a 450 alumnos que cursan el cuarto año y que estaban matriculados el año 2015 en las distintas Facultades de nuestra universidad.

La Muestra se determinó un tamaño de muestra con un nivel de confianza de $95 \%$, un error absoluto de estimación de 0.05 , asumiendo una proporción de estudiantes con una valoración de tener una buena posición económica o de trabajar en algo relacionado con su profesión de 0.80 , según la siguiente fórmula de tamaño de muestra aleatoria:

$\mathbf{n}=\frac{Z \alpha^{2} P(1-P)}{E^{2}}$

\section{Dónde:}

P: proporción de valoración de tener una buena posición económica o de trabajar en algo relacionado con su profesión de 0.80 .

Z $\alpha$ : Nivel de confianza del 95\% (1.96)

E: Error absoluto de estimación (0.05).

Reemplazando los valores, se obtiene el tamaño mínimo de muestra de 226, considerando algunas pérdidas por respuestas o cuestionarios inválidos, asumimos un $10 \%$ adicional, considerando la muestra final de 250 .

\section{RESULTADOS}

E1 63.6\% de los alumnos del estudio tiene entre 20 y 21 años, seguido por el $18.2 \%$ de alumnos que tienen mayor edad, todos los alumnos se encuentran en el cuarto año de estudios. La mayoría de alumnos son mujeres (81\%), porque incluimos a las estudiantes de Enfermería que dentro de su especialidad son más del $90 \%$, pero dentro de la especialidad de Biología y Farmacia el 34\% son varones y el $66 \%$ son mujeres; aquí recordemos que son los últimos alumnos mencionados quienes más interés manifiestan en ejercer su profesión y por ello los analizaremos con detenimiento y presentaremos sus datos en las líneas siguientes:

El lugar de nacimiento de sus padres es (el padre $50 \%$ y la madre $48 \%$ ) tanto fuera de Lima, que es la capital del Perú y queda en la costa peruana, como de fuera de Lima.

E175.8\%delos padres tiene educación superior y lo mismo sucede con el $51.5 \%$ de las madres. E1 $44 \%$ de los alumnos de Biología y Farmacia señalan que han salido a provincias del Perú de vacaciones en los últimos 5 años, el 36\% tanto a provincias del Perú como al extranjero y el $18 \%$ sólo al extranjero; siendo el promedio de salidas al extranjero de todos los alumnos encuestados de las diversas Facultades sólo $11.7 \%$.

Al respecto $57.6 \%$ de los alumnos de Biología y Farmacia están totalmente de acuerdo con la importancia que tiene ayudar a la gente que te rodea y el $36.4 \%$ está sólo de acuerdo con esto;

La mayor parte de alumnos señala (42.4\%) que están sólo parcialmente de acuerdo con querer mucho dinero y cosas caras.

Características de la personalidad poco tradicional de los alumnos de Biología y Farmacia:

a. Respecto a la importancia de lo religioso, sólo el $43.1 \%$ lo señala, en contraste al $71.9 \%$ de los estudiantes de enfermería y el $73.9 \%$ de los estudiantes de Estomatología, quienes sí le dan mucha importancia.

b. Sólo el $39.4 \%$ dice tener apego al orden y el $42.4 \%$ a obedecer.

c. Sobre la importancia que tiene mantener las costumbres, el $60.6 \%$ de los alumnos de Biología y Farmacia dicen que están de acuerdo, en cambio el $81.4 \%$ de los alumnos de Tecnología médica y el $84.6 \%$ de los alumnos de Estomatología dicen que están de acuerdo. 
d. Sobre el estar de acuerdo con evitar cualquier cosa que la gente considere incorrecta, la mayoría de los alumnos de Biología y Farmacia (39.4\%) consideran estar sólo parcialmente de acuerdo.

e. El $36.4 \%$ señala que las relaciones homosexuales pueden darse en alguna ocasión y $33.3 \%$ están de acuerdo con que siempre se den dichas relaciones; el $42.4 \%$ se muestra de acuerdo con el matrimonio homosexual y el $36.4 \%$ considera que puede darse en alguna ocasión.

f. El $48.5 \%$ de los alumnos de biología y farmacia se muestran de acuerdo con el divorcio y el $39.4 \%$ dice que se puede dar en alguna ocasión.

g. E1 $75.8 \%$ de alumnos se muestran de acuerdo con el uso de anticonceptivos y el $21.2 \%$ dicen que en alguna ocasión puede ser.

h. El $42.4 \%$ dice que el consumo de drogas puede ser en alguna ocasión y el $57.6 \%$ está en desacuerdo.

i. Respecto a las actividades religiosas el $66 \%$ de ellos no han participado en ninguna actividad religiosa en los últimos 15 días, es decir son menos participativos en lo religioso que el promedio de alumnos de la universidad.

j. Respecto a la importancia que tiene mantener el orden social, 80 alumnos de un total de 226 dicen que están de acuerdo, pero los alumnos que dicen estar sólo parcialmente de acuerdo con esto, son los que estudian Biología y Farmacia (33.3\%), Tecnología Médica (34.9\%) y Veterinaria (61.9\%).

Características de la personalidad vinculadas a la creatividad y apertura al cambio de los alumnos de Biología y Farmacia:

a. Ellos muestran aprecio por un valor universal como es entender toda clase de cosas, así el 45.5\% está de acuerdo con su importancia y el $36.4 \%$ señala que está totalmente de acuerdo con esto.

b. El $42.4 \%$ de los alumnos señala que están de acuerdo en experimentar cosas nuevas y el 33.3\% muestra estar totalmente de acuerdo con esto. c. El $39.4 \%$ señala estar de acuerdo con hacer cosas que resulten placenteras y en la misma proporción señalan que están totalmente de acuerdo.

d. Sobre la importancia que tiene lograr una vida llena de emociones, el $39.4 \%$ de los alumnos señala que están de acuerdo y el $33.3 \%$ que están totalmente de acuerdo;

e. Los alumnos muestran acuerdo con arriesgarse en un $51.5 \%$ y el $9.1 \%$ está totalmente de acuerdo.

f. Los alumnos de Biología y Farmacia también se muestran de acuerdo con consentirse a sí mismo en un $54.5 \%$ y el $42.4 \%$ señalan que están totalmente de acuerdo.

Características de la personalidad vinculadas a la autodirección y autopromoción en los alumnos de Biología y Farmacia:

a. Son los alumnos de Biología y Farmacia (39.4\%), como los que estudian Veterinaria (52.4\%), los que le dan menos importancia a tener una familia; señalando que sólo les interesa de manera regular en mayor porcentaje.

b. La mayoría de alumnos señala que lo más importante es desarrollarse como persona (140 de 226), siendo los alumnos de Biología y Farmacia los que responden en mayor medida de esta forma (72.7\%).

c. Existe una relación estadísticamente significativa entre la facultad de estudio del alumno y el grado de acuerdo con ser obediente. La mayoría de alumnos señala que está de acuerdo con ser obediente (84 de 226 alumnos), pero son los alumnos de Biología y Farmacia (45.5\%) como de Psicología (48\%), los que señalan que están sólo parcialmente de acuerdo en esto.

d. Sobre entregarse a las personas cercanas, la mayoría señala en un $39.4 \%$ que está sólo parcialmente de acuerdo, el $24.2 \%$ de acuerdo y el $9.1 \%$ totalmente de acuerdo. 
e. Señalan que en los últimos 15 días el 56.9\% no ha participado en asociación alguna, lo cual está por debajo del promedio de participación del total de alumnos encuestados.

f. El $63.6 \%$ señalan que están totalmente de acuerdo con hacer las cosas de manera original y propia, el $30.3 \%$ se muestra sólo de acuerdo con esto. g. E1 87.9\% muestran que están totalmente de acuerdo en tomar sus propias decisiones.

Como parte central de nuestro estudio presentaremos algunas Tablas que son esenciales para comprender el problema de análisis:

Tabla 1: Importancia que le dan a trabajar en algo relacionado con los estudios profesionales que hace, por facultad

\begin{tabular}{|c|c|c|c|c|c|c|c|c|c|c|c|c|c|}
\hline \multirow[t]{2}{*}{$\begin{array}{l}\text { Grado de } \\
\text { importancia }\end{array}$} & \multicolumn{2}{|c|}{$\begin{array}{l}\text { Biología- } \\
\text { Farmacia }\end{array}$} & \multicolumn{2}{|c|}{$\begin{array}{c}\text { Tecnología } \\
\text { Médica }\end{array}$} & \multicolumn{2}{|c|}{ Estomatología } & \multicolumn{2}{|c|}{ Psicología } & \multicolumn{2}{|c|}{ Veterinaria } & \multicolumn{2}{|c|}{ Enfermería } & \multirow[t]{2}{*}{ Total } \\
\hline & $\mathrm{N}^{\circ}$ & $\%$ & $\mathrm{~N}^{\circ}$ & $\%$ & $\mathrm{~N}^{\circ}$ & $\%$ & $\mathrm{~N}^{\circ}$ & $\%$ & $\mathrm{~N}^{\circ}$ & $\%$ & $\mathrm{~N}^{\circ}$ & $\%$ & \\
\hline Poco & 0 & 0.0 & 0 & 0.0 & 2 & 3.8 & 1 & 4.0 & 0 & 0.0 & 0 & 0.0 & 3 \\
\hline Regular & 0 & 0.0 & 1 & 2.3 & 1 & 1.9 & 2 & 8.0 & 1 & 4.8 & 3 & 5.8 & 8 \\
\hline Bastante & 8 & 24.2 & 13 & 30.2 & 11 & 21.2 & 5 & 20.0 & 7 & 33.3 & 26 & 50.0 & 70 \\
\hline Mucho & 25 & 75.8 & 29 & 67.4 & 38 & 73.1 & 17 & 68.0 & 13 & 61.9 & 23 & 44.2 & 145 \\
\hline Total & 33 & 100.0 & 43 & 100.0 & 52 & 100.0 & 25 & 100.0 & 21 & 100.0 & 52 & 100.0 & 226 \\
\hline
\end{tabular}

Vemos que son los alumnos de Biología y Farmacia son los que le dan más importancia a trabajar en algo relacionado a su profesión, son también ellos los que le dan más importancia a trabajar en rubros interesantes, aunque no sean de su profesión $(78.8 \%$ del total de alumnos de Biología y Farmacia).

Tabla 2: Lugar de residencia por facultad de estudio

\begin{tabular}{|c|c|c|c|c|c|c|c|c|c|c|c|c|c|}
\hline \multirow[b]{2}{*}{ Distrito en el que vive } & \multicolumn{2}{|c|}{$\begin{array}{l}\text { Biología- } \\
\text { Farmacia }\end{array}$} & \multicolumn{2}{|c|}{$\begin{array}{l}\text { Tecnología } \\
\text { Médica }\end{array}$} & \multicolumn{2}{|c|}{ Estomatología } & \multicolumn{2}{|c|}{ Psicología } & \multicolumn{2}{|c|}{ Veterinaria } & \multicolumn{2}{|c|}{ Enfermería } & \multirow[t]{2}{*}{ Total } \\
\hline & $\mathrm{N}^{\circ}$ & $\%$ & $\mathrm{~N}^{\circ}$ & $\%$ & $\mathrm{~N}^{\circ}$ & $\%$ & $\mathrm{~N}^{\circ}$ & $\%$ & $\mathrm{~N}^{\circ}$ & $\%$ & $\mathrm{~N}^{\circ}$ & $\%$ & \\
\hline $\begin{array}{l}\text { Sector A- Miraflores, La Molina, } \\
\text { Monterrico, San Borja, San Isidro, Surco }\end{array}$ & 11 & 33.3 & 1 & 2.3 & 25 & 48.1 & 12 & 48.0 & 5 & 23.8 & 1 & 1.9 & 55 \\
\hline $\begin{array}{l}\text { Sector B- Barranco, Jesús María, } \\
\text { Lince, Magdalena del Mar, Pueblo } \\
\text { Libre, San Miguel }\end{array}$ & 18 & 24.2 & 5 & 11.6 & 9 & 17.3 & 1 & 4.0 & 1 & 4.8 & 0 & 0.0 & 24 \\
\hline Sector C- Los Olivos & 1 & 3.0 & 3 & 7.0 & 6 & 11.5 & 0 & 0.0 & 4 & 19.0 & 7 & 13.5 & 21 \\
\hline Sector D- Surquillo & 0 & 0.0 & 1 & 2.3 & 0 & 0.0 & 2 & 8.0 & 0 & 0.0 & 0 & 0.0 & 3 \\
\hline $\begin{array}{l}\text { Sector E-Callao, Bellavista, La } \\
\text { Perla, Carmen de la Legua, } \\
\text { Ventanilla, Ancón }\end{array}$ & 3 & 9.1 & 3 & 7.0 & 4 & 7.7 & 1 & 4.0 & 2 & 9.5 & 3 & 5.8 & 16 \\
\hline $\begin{array}{l}\text { Sector F-San Luis, Santa Anita, } \\
\text { Chorrillos, Ate }\end{array}$ & 1 & 3.0 & 5 & 11.6 & 2 & 3.8 & 1 & 4.0 & 1 & 4.8 & 5 & 9.6 & 15 \\
\hline Sector G-Lima Cercado, Breña & 1 & 3.0 & 4 & 9.3 & 0 & 0.0 & 1 & 4.0 & 4 & 19.0 & 7 & 13.5 & 17 \\
\hline $\begin{array}{l}\text { Sector H- Carabayllo, Independencia, } \\
\text { La Victoria, Puente Piedra, San Juan } \\
\text { de Lurigancho, San Juan de Miraflores, } \\
\text { San Martín de Porres, Villa María del Triunfo }\end{array}$ & 7 & 21.2 & 19 & 44.2 & 6 & 11.5 & 7 & 28.0 & 3 & 14.3 & 24 & 46.2 & 66 \\
\hline Otro & 1 & 3.0 & 2 & 4.7 & 0 & 0.0 & 0 & 0.0 & 1 & 4.8 & 5 & 9.6 & 9 \\
\hline
\end{tabular}

Pearson $\operatorname{chi}^{2}(40)=111.6037 \quad \operatorname{Pr}=0.000$ 
Aymé Buitrón y Luz Carbajal: La vocación profesional, los valores y el proyecto de vida ...

El sector A es de ingreso económico alto o medio alto. El sector B es de ingreso medio o medio alto. El sector $\mathrm{C}$ es de ingreso medio alto, pero también de ingresos inferiores. El sector D, E y F son de ingreso medio y medio bajo. El sector $\mathrm{H}$ es de ingreso medio y pobres.

Tabla 3: Clase social de pertenencia de los alumnos de biología y farmacia por preferencia en trabajar en algo relacionado a su profesión

\begin{tabular}{|c|c|c|c|c|c|c|c|c|c|c|}
\hline \multirow{2}{*}{$\begin{array}{l}\text { Trabajar en algo } \\
\text { relacionado con } \\
\text { sus estudios o } \\
\text { profesión }\end{array}$} & \multicolumn{2}{|c|}{ Clase Baja } & \multicolumn{2}{|c|}{ Clase media baja } & \multicolumn{2}{|c|}{ Clase media } & \multicolumn{2}{|c|}{ Clase alta } & \multicolumn{2}{|c|}{ Total } \\
\hline & $\mathrm{N}^{\circ}$ & $\%$ & $\mathrm{~N}^{\circ}$ & $\%$ & $\mathrm{~N}^{\circ}$ & $\%$ & $\mathrm{~N}^{\circ}$ & $\%$ & $\mathrm{~N}^{\circ}$ & $\%$ \\
\hline Poco & 1 & 1.96 & 0 & 0.00 & 0 & 0.00 & 0 & 0.00 & 1 & 1.96 \\
\hline Regular & 0 & 0.00 & 0 & 0.00 & 2 & 3.92 & 0 & 0.00 & 2 & 3.92 \\
\hline Bastante & 0 & 0.00 & 0 & 0.00 & 7 & 13.73 & 5 & 9.80 & 12 & 23.53 \\
\hline Mucho & 1 & 1.96 & 3 & 5.88 & 21 & 41.18 & 11 & 21.57 & 36 & 70.59 \\
\hline Total & 2 & 3.92 & 3 & 5.88 & 30 & 58.82 & 16 & 31.37 & 51 & 100.00 \\
\hline
\end{tabular}

Pearson $\operatorname{chi}^{2}(9)=27.9792 \operatorname{Pr}=0.001$

Vemos la relación estadísticamente significativa entre la clase social y la preferencia por trabajar en algo relacionado con la profesión que estudian.

Tabla 4: Relación entre la importancia que dan los alumnos de Biología y Farmacia a tener una pareja y trabajar en algo relacionado con sus estudios profesionales

\begin{tabular}{|c|c|c|c|c|c|c|c|c|c|c|c|c|}
\hline \multirow{3}{*}{$\begin{array}{l}\text { Importancia que } \\
\text { dan a trabajar en } \\
\text { estudios } \\
\text { profesionales } \\
\text { que cursan }\end{array}$} & \multicolumn{12}{|c|}{ Importancia que dan los alumnos de Biología y Farmacia a tener pareja } \\
\hline & \multicolumn{2}{|c|}{ Nada } & \multicolumn{2}{|c|}{ Poco } & \multicolumn{2}{|c|}{ Regular } & \multicolumn{2}{|c|}{ Bastante } & \multicolumn{2}{|c|}{ Mucho } & \multicolumn{2}{|c|}{ Total } \\
\hline & $\mathrm{N}^{\circ}$ & $\%$ & $\mathrm{~N}^{\circ}$ & $\%$ & $\mathrm{~N}^{\circ}$ & $\%$ & $\mathrm{~N}^{\circ}$ & $\%$ & $\mathrm{~N}^{\circ}$ & $\%$ & $\mathrm{~N}^{\circ}$ & $\%$ \\
\hline Poco & 0 & 0.00 & 1 & 1.96 & 0 & 0.00 & 0 & 0.00 & 0 & 0.00 & 1 & 1.96 \\
\hline Regular & 0 & 0.00 & 0 & 0.00 & 1 & 1.96 & 0 & 0.00 & 1 & 1.96 & 2 & 3.92 \\
\hline Bastante & 1 & 1.96 & 0 & 0.00 & 8 & 15.69 & 3 & 5.88 & 0 & 0.00 & 12 & 23.53 \\
\hline Mucho & 3 & 5.88 & 1 & 1.96 & 16 & 31.37 & 13 & 25.49 & 3 & 5.88 & 36 & 70.59 \\
\hline Total & 4 & 7.84 & 2 & 3.92 & 25 & 49.02 & 16 & 31.37 & 4 & 7.84 & 51 & 100.00 \\
\hline
\end{tabular}

Pearson $\operatorname{chi}^{2}(12)=32.7817 \operatorname{Pr}=0.001$

Apreciamos la relación estadísticamente significativa entre la importancia que tiene trabajar en sus estudios profesionales y el tener una pareja. En tanto estudiamos el tema de la vocación profesional, debemos detenernos a analizar más el perfil de los alumnos que tienen mayor interés en ejercer su profesión, es decir comprender más el caso de los alumnos de Biología y Farmacia; respecto a esto último, vemos que la mayoría de ellos responde que el tener una pareja es sólo de interés medio o regular. 


\section{DISCUSIÓN}

Nosotros podemos comparar nuestro grupo de estudio con los Millennials de Argentina (Cuesta, Ibáñez, Tagliabue y Zangaro 2009), a ellos se les pregunta sobre el lugar que ocupa el trabajo en sus vidas y el $55.3 \%$ señala que lo considera un ámbito de crecimiento personal, lo que coincide con la vocación por ejercer la profesión de los alumnos que estudiamos, ya que además en Argentina señalan que consideran el trabajo como un medio para su propio desarrollo.

E1 $62.75 \%$ de los estudiantes de Biología y Farmacia se auto clasifica de clase media o media alta, lo que se corrobora con la información sobre el distrito en el que viven (Tabla 2). En la Tabla 3 vemos la relación estadísticamente significativa entre la clase social y la preferencia por trabajar en algo relacionado con la profesión que estudian. Esto se puede explicar porque los alumnos con menos confort económico tienen una mayor urgencia por trabajar, aunque no sea en su profesión; además el gran deseo por trabajar en la profesión elegida se puede vincular a la formación profesional que tiene la mayoría de padres de los estudiantes en cuestión, quienes por la tranquilidad económica y ejemplo de vida han transmitido su vocación profesional.

Sobre los Millennialls en España, los estudios indican que son empleados muy exigentes respecto a lo que esperan de un trabajo sobre todo porque tienen detrás una familia que los respaldará económicamente, si deciden abandonarlo en caso de que no responda a sus expectativas. (Cuesta, Ibáñez, Tagliabue y Zangaro 2009). Creemos que es por lo antes mencionado que los Millennials peruanos de nuestro estudio son semejantes a los Millennials españoles, ya que el confort económico de los padres influye mucho en su modo de vida y expectativas de ejercicio profesional.

Los alumnos de Biología y Farmacia señalan que el aspecto más importante en su vida es desarrollarse como personas y consideran sólo de manera regular, la importancia de ganar dinero. En este aspecto los alumnos de Biología y Farmacia no coinciden con los Millennials peruanos de otros estudios, ya que en ellos se encuentra que el dinero y la estabilidad laboral sí interesa a la mayoría de ellos (Begazo y Fernández Baca, 2015).
La mayoría de los alumnos de Biología y Farmacia muestran apertura al cambio y no son tradicionales, ya que ellos no consideran importante tener hijos ni la religión, además la mayoría está de acuerdo con el matrimonio y las relaciones homosexuales y el uso de anticonceptivos. Respecto a la poca importancia que tiene para los jóvenes de nuestro estudio el tener hijos, encontramos coincidencias con los Millennials de España (Emailingnetwork, 2014), ya que ellos encuentran quesonlosjóvenescon estudios de doctorado quienes menos hijos tienen, también se encuentra que esta tendencia está relacionada con la prolongación de los años de estudio y su estilo de vida. Esta última característica muestra a dichos jóvenes como poco tradicionales, lo que se confirma con la poca importancia que le dan a ser obedientes, ya que en primer lugar señalan estar sólo parcialmente de acuerdo con esto y en segundo lugar dicen lo mismo los alumnos de Psicología; en cambio los alumnos de Estomatología muestran mayor acuerdo con ser obedientes y en segundo lugar los alumnos de Enfermería. Aquí podemos concluir en que los alumnos de Biología y Farmacia se muestran poco tradicionales y bastante auto dirigidos. Pero la apertura al cambio de los estudiantes de Biología y Farmacia no es total, ya que la mayoría está en desacuerdo con el aborto; el $51.5 \%$ de estudiantes de Biología y Farmacia se muestra algo tradicional al manifestar su desacuerdo con el aborto, de tal manera que el $30.3 \%$ dice que es posible solo en alguna ocasión y $18.2 \%$ sí están de acuerdo.

En el Perú la cultura tradicional no ha desaparecido y sus características culturales se encuentran en gran parte de la población y también entre los jóvenes, pero dichas características no son centrales en el estilo de vida y valores de los estudiantes de Biología y Farmacia de la Universidad Peruana Cayetano Heredia. La perspectiva de cambio es mayor entre los Millennials argentinos, quienes en un $53.2 \%$ dicen estar a favor del aborto y el $78.6 \%$ a favor del matrimonio entre personas del mismo sexo (Barómetro- opinión pública e investigación de mercado, 2013).

Las condiciones de competencia internacional, desplegadas desde los años 90 del siglo 20 en Perú, han contribuido al desarrollo de una estructura social y de trabajo muy competitiva, es en este contexto 
Aymé Buitrón y Luz Carbajal: La vocación profesional, los valores y el proyecto de vida ...

que los jóvenes en la actualidad ya no son los mismos que décadas anteriores y el individualismo toma cada vez más espacio en sus vidas (Cuesta, Ibáñez, Tagliabue y Zangaro, 2009).

Hemos observado en los datos antes presentados que los estudiantes de Biología y Farmacia en su mayoría están totalmente de acuerdo con demostrar lo capaz que son, tomar sus propias decisiones, tener una vida llena de emociones, hacer cosas que resulten placenteras, consentirse a sí mismos, mucho interés en entender toda clase de cosas y experimentar cosas nuevas; de lo que podemos concluir que un grupo mayoritario se orienta hacia el desafío, la creatividad, el entusiasmo, la curiosidad y la independencia, características que son muy importantes para los profesionales y en todo investigador (Schwartz, 1999). Esto coincide con los estudios de los Millennials que concluyen en que ellos ponderan el presente por sobre todas las cosas (Begazo y Fernandez Baca, 2015).

\section{CONCLUSIÓN}

Los alumnos de Biología y Farmacia son los que muestran mayor interés en trabajar en su profesión. Ellos son hijos de profesionales en su mayoría, a diferencia de otros Millennials no muestran mucho interés en las ganancias materiales durante su vida futura. Su origen de clase social media o alta, condiciona en parte su falta de interés en trabajar en ocupaciones que no estén vinculadas a su profesión, ya que su urgencia económica es menor que la de grupos con menores recursos económicos. Ellos son poco tradicionales, curiosos, creativos, auto direccionados, no le temen al riesgo, quieren entender toda clase de cosas, creativos, se auto promocionan y tienen apertura al cambio, pero la mayoría no apoya el aborto en cualquier circunstancia.

\section{REFERENCIAS}

Barómetro- opinión pública e investigación de mercado (2013). Millennials- Estudio de valores y prácticas de jóvenes argentinos [En Línea]. Recuperado 29/8/2016 desde http:// www.ibarometro.com/newsite/wp-content/ uploads /2014/11/Informe-MillennialsArgentinos.pdf

Begazo Villanueva, J., y Fernández Baca, W. (2015). Los millennials peruanos: características y proyecciones de vida. En: Gestión en el Tercer Milenio, Revista de Investigación de la Facultad de Ciencias Administrativas, UNMSM, Vol. 18-II (36), 9-15.

CEPLAN (2015), Millennials marcarán la fuerza laboral en el Perú dentro de 10 años. En: Diario Gestión. [En línea]. Recuperado 4/3/2015 desde http://gestion.pe/economia/millennialsmarcaran-fuerza-laboral-peru-dentro-10-anos2125177

Ciochina, L., Faria, L. (2011). "Padroes de associacao entre individualismo-colectivismo e concepcoes pessoais de inteligencia nos contextos culturais portugués e romeno". En: Libro de actas del XI Congreso Internacional Galego-Portugués de Psicopedagoxía (2012-2019). España: Universidad da Coruña.

Cuesta, E., Ibañez, M., Tagliabue, R y Zangaro, M. (2009). "La nueva generación y el trabajo". En Barbarói (126-138). Recuperado 11/7/ 2016 desde https://online.unisc.br/seer/index.php/ barbaroi/article/viewFile/479/908

Emailingnetwork (2014). Radiografia de la Generación del Milenio en España - Estudio sobre las principales preocupaciones y consumo de ocio de los jóvenes de entre 18 y 34 años [En línea]. Recuperado 3/10/2016 desde https://emailingnetwork. com/wp-content/uploads/pdf/Millenialsestudio-EmailingNetwork.pdf

Hofstede, G. (1991). Culture and organizations: software of the mind. London: McGraw-Hill.

Laloup, J. (1968). Progreso moral o decadencia de la costumbre. Madrid: Fax.

Manpower Group (2016), Carreras profesionales de los Millennials: Horizonte 2020 Hechos, cifras y consejos prácticos de los expertos en empleo [En línea]. Recuperado 19/9/2016 desde http://www. manpowergroup.es/data/files/Estudios/ pdf/Estudio_ManpowerGroup_-_Carreras_ profesionales_de_los_Millennials,_Horizonte_ 2020_635997053700335000.pdf 
Maslow, A. (1954). Motivation and personality, New York: Harper and Row.

Molinari, P. (2011). Turbulencia Generacional. Buenos Aires: Temas Grupo Editorial.

Omar, A. (2006). Justicia Organizacional, individualismo-colectivismo y estrés laboral, En: Psicología y Salud, Universidad Veracruzana, Vol. 16 (2), 207-217.

Pantoja, C. (1992), "En torno al concepto de vocación", En: Educación y Ciencia, Vol. 2 (6), 17-20.

Parsons, T. (1951). El sistema social. Cambridge: Mass.

Penagos, T. y Rubio, E. (2015). Millennials y Millennials peruanos - Realidad, expectativas y proyecciones[Enlínea]. Recuperado28/9/2016 desde http://talentnewsla.com/white-papermillennials-y-millennials-peruanos-realidadexpectativas-y-proyecciones/

Schwartz, S. (1999). "A theory of cultural values and some implications for work", En: Revista Internacional de Psicología Aplicada, 48, 2347.

Triandis,H.(1994). "Theoreticalandmethodological approaches to the study of collectivism and individualism". En: Kim, U., Triandis, H., Kagitcibasi C., Choi, S., Yoon, G. editors, Individualism and collectivism: Theory, method, and applications (41-51). Estados Unidos: Sage Publications. 